\title{
Exercise medicine in cancer care
}

Prue Cormie, Mark Trevaskis, Elysia Thornton-Benko, Eva M Zopf

\section{Background}

Every four minutes, an Australian is diagnosed with cancer. Early detection and effective treatment means that many of these people are living with the side effects of cancer and its treatment for numerous years.

\section{Objective}

The aim of this article is to summarise the evidence examining the role of exercise in cancer care.

\section{Discussion}

Exercise is a safe and effective adjunct therapy in cancer care. Patients with cancer who regularly engage in moderate-intensity exercise are more likely to have: fewer and less severe treatment-related side effects; a lower relative risk of developing other chronic diseases; and, in some cases, a lower relative risk of cancer recurrence and mortality. Available evidence highlights the benefits of general practitioners (GPs) discussing and recommending exercise to their patients with cancer. To optimise the therapeutic effect of exercise, GPs may consider referring patients with cancer to an exercise physiologist or physiotherapist who has experience in cancer care.
ONE IN TWO AUSTRALIANS will be diagnosed with cancer by the age of 85 years. ${ }^{1}$

Cancer and its treatment can cause serious health issues that compromise physical and mental health and wellbeing. Cancer is responsible for the highest burden of disease in Australia; consequently, current health services have difficulty adequately managing these issues. ${ }^{2}$ Evidence from a significant body of preclinical, clinical trial and epidemiological research has established exercise as a safe and effective adjunct therapy in cancer care. ${ }^{3-5}$ This level of evidence has led major health organisations across the world to identify the need to incorporate exercise as part of standard practice in cancer care. ${ }^{6-12}$ This article summarises the evidence base for these recommendations.

\section{Key definitions}

- Cancer: A term for diseases in which abnormal cells divide without control and can invade nearby tissues. Cancer cells can also spread to other parts of the body through the blood and lymph systems. There are several main types of cancer. Carcinoma is a cancer that begins in the skin or in tissues that line or cover internal organs. Sarcoma is a cancer that begins in bone, cartilage, fat, muscle, blood vessels or other connective or supportive tissue.

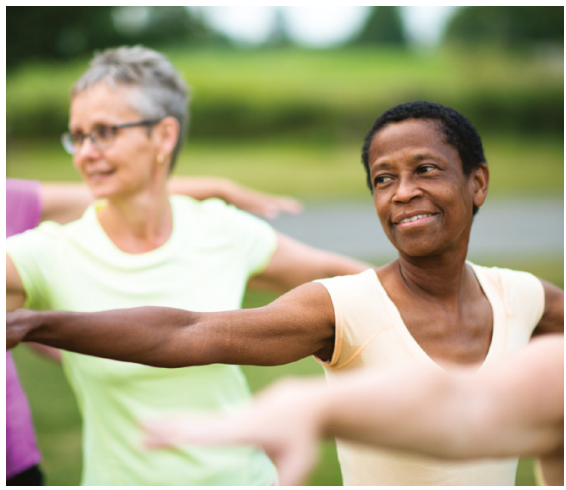

Leukaemia is a cancer that starts in blood-forming tissue, such as the bone marrow, and causes large numbers of abnormal blood cells to be produced and enter the blood. Lymphoma and multiple myeloma are cancers that begin in the cells of the immune system. Central nervous system cancers are cancers that begin in the tissues of the brain and spinal cord. Cancer is also called 'malignancy'.

- Exercise: A type of physical activity consisting of planned, structured and repetitive bodily movement for the purpose of improving and/or maintaining health and physical fitness.

- Aerobic exercise: A form of exercise that predominately stresses the cardiovascular system. This refers to any exercise that uses large muscle groups, can be maintained continuously and is rhythmical in nature (eg walking, jogging, cycling and swimming). It is also referred to as 'cardiovascular exercise' or 'endurance exercise'.

- Resistance exercise: A form of exercise that predominately stresses the musculoskeletal system. This refers to exercise that requires a muscle or a muscle group to work against external resistance (eg squats, chair rises, chest press and push ups). It is also referred to as 'muscle strengthening exercise' or 'weight training'. 


\section{Exercise attenuates cancer} treatment-related side effects

Hundreds of clinical trials synthesised in more than 40 meta-analyses across various cancer types, stages and treatment regimens show that exercise can help counteract the negative effects of cancer and its treatment (Figure 1)..$^{3-5}$ The level of evidence now available clearly suggests that withholding exercise from patients with cancer is harmful. Specifically, patients with cancer who do not exercise have a higher degree of functional impairment, worse cancer-related fatigue, more psychological distress and a poorer quality of life. ${ }^{3-12}$
Strong evidence confirms that high-quality exercise significantly reduces the most common treatment-related side effects. ${ }^{3-12}$ Exercise has been established as the most effective first-line treatment for cancer-related fatigue, significantly reducing its presence and severity. ${ }^{13}$ Exercise counteracts the physical deconditioning caused by cancer and its treatment, significantly improving physical function and making everyday activities easier to undertake. ${ }^{14}$ Exercise leads to significant reductions in the psychological distress associated with cancer, alleviating depressive symptoms and anxiety. ${ }^{15}$

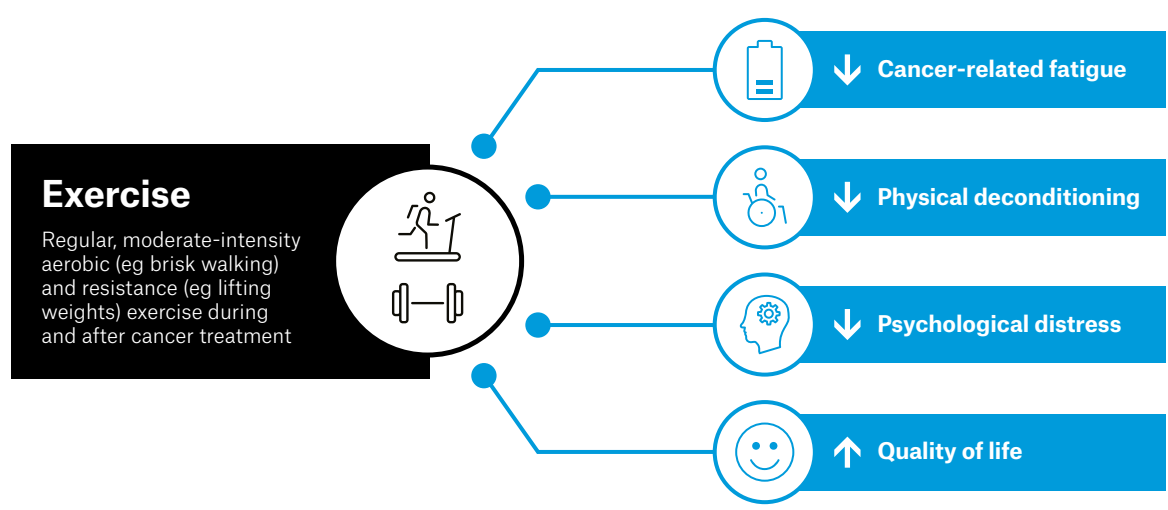

Figure 1. Exercise effectively counteracts the most common side effects of cancer and its treatment. ${ }^{3-16}$

\section{Exercise observed to reduce the relative risk of:}

\section{CANCER RECURRENCE}

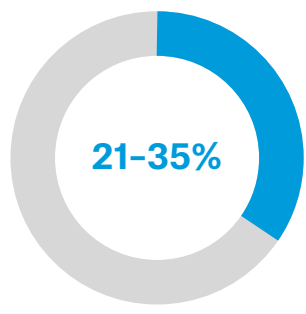

Hazard ratios from

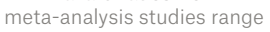

meta-analysis studies range

to $0.65[0.56-0.75](n=38,560)$
CANCER MORTALITY

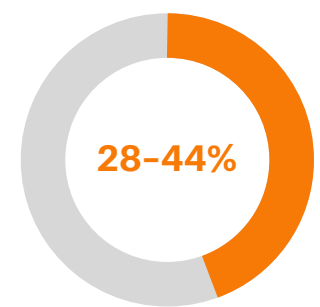

Hazard ratios from

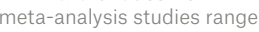

from $0.72[0.60-0.85](n=21,382)$

to $0.56[0.38-0.83](n=10,470)$
ALL-CAUSE MORTALITY



Hazard ratios from

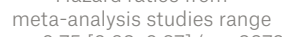

from $0.75[0.62-0.87](\mathrm{n}=2379)$ to $0.52[0.43-0.64](n=21,647)$

Figure 2. Exercise confers a protective effect against cancer recurrence, cancer-specific mortality and all-cause mortality in some cancers (data arises from studies involving predominately patients with breast, colorectal and prostate cancer). ${ }^{3}$

Patients with cancer who exercise report a significantly greater quality of life across multiple cancer-specific and general health domains. ${ }^{16}$

There is a growing body of evidence to indicate that appropriately prescribed exercise may also help manage and alleviate other cancer-related symptoms and side effects. Exercise may help maintain a healthy balance of muscle and fat mass, preserve bone mass, manage lymphoedema, enhance sleep quality, decrease pain, counteract peripheral neuropathy, attenuate cardiotoxicity, improve cognitive function (ie mitigate chemo-fog/chemo-brain) and enhance sexual wellbeing. ${ }^{3,6,17}$ Exercise also appears to reduce the risk of patients with cancer developing other chronic diseases that may be exacerbated by cancer and its treatment, such as cardiovascular disease, diabetes and osteoporosis. . $^{3,6,17}$

\section{Exercise may affect cancer progression}

Data from more than 35 observational studies suggest that exercise confers a protective effect against cancer-specific mortality, cancer recurrence and all-cause mortality in some cancers. ${ }^{3}$ These data show that patients who exercise regularly have a $21-35 \%$ lower relative risk of cancer recurrence, a $28-44 \%$ reduced relative risk of cancer-specific mortality and a $25-48 \%$ decreased relative risk of all-cause mortality when compared with patients who do little or no exercise (Figure 2). ${ }^{3}$ Most evaluations to date have involved patients with breast, colorectal or prostate cancer, so it is unclear whether exercise is associated with improved disease outcomes in patients diagnosed with other cancer types.

Given the nature of epidemiological investigations, this research is limited by the inability to infer direct causality between exercise behaviour and cancer outcomes. Thus, it is possible that observations of the protective effect of exercise may reflect reverse causality rather than a physiological effect. That is, better outcomes may be reported for more active patients because they are less encumbered by advanced or aggressive 
disease and/or severe symptomology rather than as a result of exercise-induced adaptations that slow cancer progression. However, the protective effect of exercise was observed in multivariable adjusted analyses that accounted for a range of clinically relevant covariates associated with cancer progression such as cancer stage, treatments, smoking status, body mass index and comorbidities. Furthermore, a range of physiological adaptations that modulate tumour progression have been hypothesised to contribute to these findings. These include favourable exercise-related changes to blood perfusion and tumour vascularisation, immune function, inflammation, oxidative stress and metabolism. ${ }^{18-20}$ Preclinical investigations involving mouse models have shown that exercise in mice undergoing treatment for breast or prostate cancer led to significantly reduced tumour growth and less metastatic disease as well as improved chemotherapeutic efficacy. ${ }^{18-20}$

\section{Various mechanisms underpin the beneficial impact of exercise in cancer \\ Exercise provides such widespread benefits to people with cancer because it of cancer, various stages and prognoses and many different kinds of treatments, all of which cause a large variety of side effects. Given this complexity, a 'one size fits all' approach for exercise in cancer} is a therapy that improves the structure and function of most of the body's systems simultaneously. In fact, there is no medication or treatment that can positively influence as many body systems as exercise can. As such, a range of potential factors may contribute to the therapeutic effect of exercise for patients with cancer.

Exercise prevents and/or mitigates the negative impact of cancer and its treatment on the musculoskeletal and cardiovascular systems that contribute to physical deconditioning, cancerrelated fatigue and other symptoms. ${ }^{14}$ By enhancing functional status and reducing treatment-related side effects, exercise may allow patients to tolerate greater dosages of cancer treatment, potentially improving the treatment response. ${ }^{21}$ Regular exercise contributes to the resolution of immune homeostasis through modulating the systemic milieu like growth factor 1), inflammatory (eg C-reactive protein, interleukin 6) and sex steroid (eg oestrogen) markers. ${ }^{22}$ Exercise may enhance the effectiveness of anti-cancer treatments by normalising the tumour microenvironment and potentially increasing the transport of systemic therapies to cancer cells. ${ }^{18-20}$ Exercise potential of epigenetic modifications that phenotypic adaptations. ${ }^{23}$ Furthermore, regular exercise is an established prophylactic measure that reduces the risk of developing comorbid conditions such as heart disease, hypertension, diabetes and osteoporosis. Further research is required to elucidate the precise mechanisms underlying the therapeutic effect of exercise in cancer.

\section{Exercise is not 'one size fits all'}

Cancer is a highly complex disease that affects people throughout the lifespan. There are hundreds of different types of metabolic (eg insulin, glucose, insulinmay also enhance outcomes through the are concordant with health-enhancing

Avoid inactivity and be as care will never be appropriate. ${ }^{24}$ Similar to many cancer treatments, exercise is a therapy that has a virtually unlimited array of regimens that can be specifically tailored to counteract the negative effects of cancer. By understanding what happens to the physiology of patients with cancer during exercise, specialists can prescribe different types of exercise, at precise intensities and volumes, on the basis of a mechanism of action and dosage required to elicit a desired health outcome/adaptation. More research is required to determine the optimal exercise prescription for all types and stages of cancer. However, exercise specialists can draw on extensive evidence identifying the cardiovascular, musculoskeletal, metabolic and endocrine response to exercise derived from patients with cancer and other populations to maximise the safety and efficacy of exercise for patients with cancer.

In Australia, accredited exercise physiologists and physiotherapists are recognised as the most appropriate health professionals to prescribe and deliver exercise programs to people with cancer. Exercise physiologists and physiotherapists are tertiary-qualified allied health professionals with specialist training in exercise for patients. There

\section{Exercise prescription for all people with cancer}

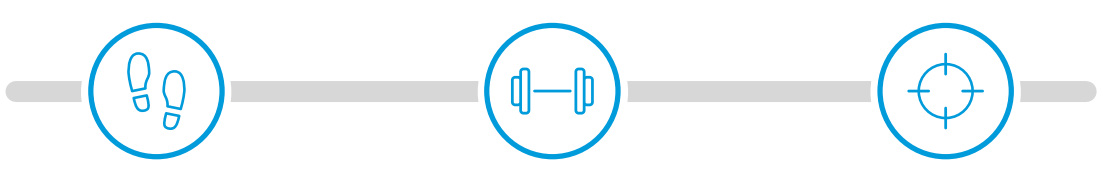
physically active as their current condition allows (ie return to normal daily activities as soon as possible after cancer diagnosis)
Tailor the exercise recommendations to the individual's abilities, noting that adaptations may be required on the basis of cancer- and treatment-related side effects, disease trajectory and current health status

Figure 3. Evidence-based exercise recommendations for people with cancer ${ }^{6-12}$ 
are over 5000 exercise physiologists and 20,000 physiotherapists currently practising throughout Australia. Their services are eligible for subsidies through the Medicare Benefits Schedule and a significant number of private health insurers. Exercise physiologists and physiotherapists pragmatically apply the available evidence while using clinical judgement and expertise to incorporate the patients' conditions, needs and preferences into an individually tailored and responsive exercise prescription. Exercise physiologists and physiotherapists who have specialist training and experience in oncology are equipped to appropriately apply screening, monitoring, programming and behaviour change strategies for the vast array of cancer types, stages and treatment options for patients of all ages who may have a range of complex cancer-related side effects and comorbid conditions. ${ }^{17}$

\section{What is the exercise prescription for patients with cancer?}

As summarised in Figure 3, the evidencebased guidelines ${ }^{6-12}$ recommend people with cancer:

- avoid inactivity and be as physically active as their current condition allows

- participate in regular moderateintensity aerobic and resistance exercise

- perform exercise tailored to the individual's abilities, noting that specific exercise programming adaptations may be required for people with cancer on the basis of disease and treatment-related adverse effects, anticipated disease trajectory and their health status.

Evidence indicates that high-quality exercise is required to elicit significant therapeutic effects in patients with cancer. ${ }^{25}$ High-quality exercise requires patients to be exercising at an intensity that is at least moderate, which equates to a rating of perceived exertion of 'somewhat hard' to 'hard'. Moderateintensity exercise should be performed regularly, with significant benefits most commonly observed in programs consisting of at least three exercise sessions weekly for 12 weeks. It is likely that a dose-response relationship exists, with higher intensities and higher volumes of exercise leading to superior reductions in symptom severity for patients with cancer. Benefits are significantly larger when exercise programs are supervised by an exercise specialist when compared with unsupervised programs. ${ }^{25}$

Clinical trial research has established that exercise is safe for people with cancer during and after cancer treatment. ${ }^{6-12}$ Patients with cancer may have exerciserelated adverse effects that are common to all people who exercise. As such, there is a possibility that exercise may result in mild discomfort, muscle soreness and muscle pulls or strains. It is less common but possible that exercise may be associated with nausea, light-headedness, fainting or falls. It is critical to highlight that safety data arise from research trials that are conducted in controlled environments (eg supervised by experienced health professionals in clinicbased environments) and typically among patients who are not experiencing severe symptoms. Consequentially, exercise for patients with cancer is safe when it is appropriately prescribed and monitored.

As a result, it is strongly recommended that patients with cancer be referred to an exercise physiologist or physiotherapist who has training and experience in cancer care. ${ }^{6,17}$ These specialists have the required training, expertise and time to appropriately apply evidence-based recommendations to maximise the safety and efficacy of exercise for patients with cancer. Referral to an exercise physiologist or physiotherapist with experience in cancer care will ensure that patients receive an individualised exercise prescription that accounts for their goals and circumstances, functional capacity, cancer stage and disease trajectory, treatment history and current treatment status, type and severity of symptoms currently experienced and anticipated, comorbid diseases and other health conditions, as well as any other factors that may influence the safety and efficacy of exercise. ${ }^{6,17}$

\section{Exercise should be prescribed as part of routine cancer care} The Clinical Oncology Society of Australia, supported by more than 25 leading health organisations, recently published a position statement calling for all health professionals involved in the care of people with cancer to incorporate exercise as a standard component of patients' cancer care plans (Figure 4$){ }^{6}$ Many challenges to integrating exercise in routine cancer care exist for both health professionals and patients with

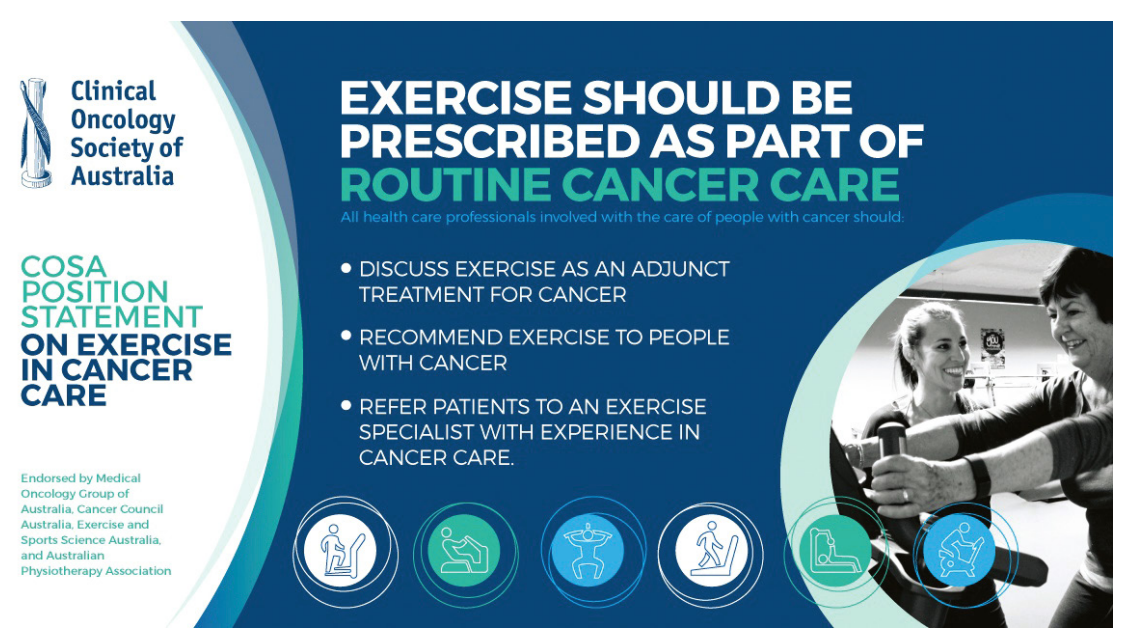

Figure 4. The Clinical Oncology Society of Australia Position Statement on Exercise in Cancer Care calls for exercise to be embedded as part of standard practice in cancer care. ${ }^{6}$

Reprinted with permission from the Clinical Oncology Society of Australia. 
cancer. However, research and extensive clinical experience identifies two key strategies general practitioners (GPs) can adopt to facilitate patients' participation in safe and efficacious exercise: discuss and recommend exercise specifically as part of the cancer care plan, and provide a referral to an exercise physiologist or physiotherapist with experience in cancer care using the appropriate Chronic Disease Management item. These actions will provide the support and expert advice required for patients to access exercise that is tailored to counteract the negative effects of their cancer.

An online directory of accredited exercise physiologists is available at the Exercise \& Sports Science Australia website (www.essa.org.au/find-aep), and a directory of physiotherapists is available at the Australian Physiotherapy Association website (https://choose. physio). Accredited cancer-specific training for exercise specialists is available at the EX-MED Cancer PD website (https://exmedcancerpd.learnbook.com. $\mathrm{au}$ ), the Breast Cancer PD website (https:// breastcancerpd.learnbook.com.au) and the Prostate Cancer PD website (https:// prostatecancerpd.learnbook.com.au).

\section{Conclusion}

When appropriately prescribed, evidence suggests that exercise has a significant therapeutic effect in the management of cancer. If people with cancer perform high-quality exercise regularly, they may significantly reduce the severity of cancer symptoms, improve their physical and mental health and wellbeing and potentially extend their survival. GPs can effectively implement exercise in cancer care by:

- discussing exercise as an adjunct therapy that effectively counteracts the adverse effects of cancer and its treatment

- recommending regular, high-quality aerobic (eg brisk walking) and resistance (eg weight lifting) exercise to people with cancer

- referring patients with cancer to an accredited exercise physiologist or physiotherapist with experience in cancer care for the exercise prescription to be individually tailored to the needs of each patient (including those with poor function and/or complex symptoms).

\section{Patient resources}

- Cancer Council - Exercise for People Living with Cancer: A guide for people with cancer, their families and friends, www.cancervic.org.au/downloads/ resources/booklets/exercise-andcancer.pdf

- Exercise \& Sports Science Australia What is the role of Accredited Exercise Physiologists in the treatment of cancer?, www.cancerwa.asn.au/resources/201904-11-ESSA-Consensus-StatementCancer-Consumers.pdf

- Exercise \& Sports Science Australia Exercise \& Cancer eBook, https://mailchi. mp/essa/exerciseandcancer

- EX-MED Cancer - Best-practice exercise medicine program for people with cancer, www.exmedcancer.org.au/

- Clinical Oncology Society of Australia Position statement on exercise in cancer care, www.cosa.org.au/publications/ position-statements.aspx

\section{Authors}

Prue Cormie PhD, AEP, Principal Research Fellow in Exercise Oncology, Mary MacKillop Institute for Health Research, Australian Catholic University, Vic. prue.cormie@acu.edu.au

Mark Trevaskis AEP, Clinical Exercise Physiologist in Exercise Oncology, Mary MacKillop Institute for Health Research, Australian Catholic University, Melbourne, Vic

Elysia Thornton-Benko BSc, MBBS (Hons), PhD, FRACGP, Specialist General Practitioner/Family Physician, Bondi Road Doctors, NSW

Eva M Zopf PhD, Postdoctoral Research Fellow in Exercise Oncology, Mary MacKillop Institute for Health Research, Australian Catholic University, Vic Competing interests: PC is the Founder and Director of EX-MED Cancer Ltd, a not-for-profit organisation that provides exercise medicine services to people with cancer. PC is the Director of Exercise Oncology EDU Pty Ltd, a company that provides fee for service training courses to upskill exercise professionals in delivering exercise to people with cancer. ETB is the Director of Wellac Lifestyle.

Funding: None.

Provenance and peer review: Commissioned, externally peer reviewed.

\section{References}

1. Australian Institute of Health and Welfare. Cancer in Australia 2019. Cancer series no.119. Cat. no. CAN 123. Canberra: AlHW, 2019.

2. Australian Institute of Health and Welfare. Burden of cancer in Australia: Australian burden of disease study 2011. Australian Burden of Disease Study series no. 12. Cat. no. BOD 13. Canberra; AlHW, 2017.

3. Cormie P, Zopf EM, Zhang X, Schmitz KH. The impact of exercise on cancer mortality, recurrence, and treatment-related adverse effects. Epidemiol Rev 2017;39(1):71-92. doi: 10.1093/epirev/mxx007.

4. Ashcraft KA, Warner AB, Jones LW, Dewhirst MW. Exercise as adjunct therapy in cancer. Semin Radiat Oncol 2019;29(1):16-24. doi: 10.1016/j. semradonc.2018.10.001.

5. Christensen JF, Simonsen C, Hojman P. Exercise training in cancer control and treatment. Compr Physiol 2018;9(1):165-205. doi: 10.1002/cphy. c180016.

6. Cormie P, Atkinson M, Bucci L, et al. Clinical Oncology Society of Australia position statement on exercise in cancer care. Med J Aust 2018;209(4):184-87. doi: 10.5694/mja18.00199.

7. National Comprehensive Cancer Network. NCCN Clinical Practice Guidelines in Oncology Survivorship. Plymouth Meeting, PA: NCCN, 2018.

8. Rock CL, Doyle C, Demark-Wahnefried W, et al. Nutrition and physical activity guidelines for cancer survivors. CA Cancer J Clin 2012;62(4):243-74. doi: 10.3322/caac.21142.

9. ASCO Cancer Survivorship Committee. Providing high quality survivorship care in practice: An ASCO guide. Alexandria, VA: American Society of Clinical Oncology, 2014

10. Hayes SC, Newton RU, Spence RR, Galvao DA. The exercise and sports science Australia position statement: Exercise medicine in cancer management. J Sci Med Sport 2019;22(11):1175-99. doi: 10.1016/j.jsams.2019.05.003.

11. Campbell KL, Winters-Stone KM, Wiskemann $J$, et al. Exercise guidelines for cancer survivors: consensus statement from international multidisciplinary roundtable. Med Sci Sports Exerc 2019;51(11):2375-90. doi: 10.1249/ MSS.0000000000002116.

12. Campbell A, Stevinson $\mathrm{C}$, Crank H. The BASES expert statement on exercise and cancer Survivorship. J Sports Sci 2012;30(9):949-52. doi: 10.1080/02640414.2012.671953.

13. Mustian KM, Alfano CM, Heckler C, et al. Comparison of pharmaceutical, psychological, and exercise treatments for cancer-related fatigue: A meta-analysis. JAMA Oncol 2017;3(7):961-68. doi: 10.1001/jamaoncol.2016.6914.

14. Fuller JT, Hartland MC, Maloney LT, Davison K. Therapeutic effects of aerobic and resistance exercises for cancer survivors: A systematic review of meta-analyses of clinical trials. $\mathrm{Br}$ J Sports Med 2018;52(20):1311. doi: 10.1136/ bjsports-2017-098285.

15. Craft LL, Vaniterson EH, Helenowski IB, Rademaker AW, Courneya KS. Exercise effects on depressive symptoms in cancer survivors: A systematic review and meta-analysis. Cancer Epidemiol Biomarkers Prev 2012;21(1):3-19. doi: 10.1158/1055-9965.EPI-11-0634.

16. Mishra SI, Scherer RW, Geigle PM, et al. Exercise interventions on health-related quality of life for cancer survivors. Cochrane Database Syst Rev 2012;8:CD007566. doi: 10.1002/14651858. CD007566.pub2.

17. Turner J, Marthick M, Murnane A, et al. Consensus statement on the role of accredited exercise physiologists in the treatment of cancer: A guide for all health professionals involved in the care of people with cancer. Brisbane: Exercise and Sports Science Australia, 2019. 
18. Koelwyn GJ, Quail DF, Zhang X, White RM, Jones LW. Exercise-dependent regulation of the tumour microenvironment. Nat Rev Cancer 2017;17(10):620-32. doi: 10.1038/nrc.2017.78.

19. Betof AS, Dewhirst MW, Jones LW. Effects and potential mechanisms of exercise training on cancer progression: A translational perspective. Brain Behav Immun 2013;30 Suppl:S75-87. doi: 10.1016/j.bbi.2012.05.001.

20. Schadler KL, Thomas NJ, Galie PA, et al. Tumor vessel normalization after aerobic exercise enhances chemotherapeutic efficacy. Oncotarget 2016;7(40):65429-40. doi: 10.18632/ oncotarget.11748.

21. Bland KA, Zadravec K, Landry T, Weller S, Meyers L, Campbell KL. Impact of exercise on chemotherapy completion rate: A systematic review of the evidence and recommendations for future exercise oncology research. Crit Rev Oncol Hematol 2019;136:79-85. doi: 10.1016/j. critrevonc.2019.02.005.

22. Hojman P, Gehl J, Christensen JF, Pedersen BK. Molecular mechanisms linking exercise to cancer prevention and treatment. Cell Metab 2018;27(1):10-21. doi: 10.1016/j.cmet.2017.09.015.

23. Thomas RJ, Kenfield SA, Jimenez A. Exerciseinduced biochemical changes and their potential influence on cancer: A scientific review. $\mathrm{Br} J$ Sports Med 2017;51(8):640-44. doi: 10.1136/ bjsports-2016-096343.

24. Cormie P, Adams D, Atkinson M, et al. Exercise as part of routine cancer care. Lancet Oncol 2018;19(9):e432. doi: 10.1016/S14702045(18)30598-9.

25. Buffart LM, Kalter J, Sweegers MG, et al. Effects and moderators of exercise on quality of life and physical function in patients with cancer: An individual patient data meta-analysis of $34 \mathrm{RCTs}$. Cancer Treat Rev 2017;52:91-104. doi: 10.1016/j. ctrv.2016.11.010 